Proyecciones Journal of Mathematics

Vol. 37, No 1, pp. 19-43, March 2018.

Universidad Católica del Norte

Antofagasta - Chile

\title{
Vertex graceful labeling of some classes of graphs
}

\author{
A. P. Santhakumaran \\ Hindustan Institute of Technology and Science, India \\ P. Balaganesan \\ Hindustan Institute of Technology and Science, India \\ Received: July 2016. Accepted : November 2016
}

\begin{abstract}
$A$ connected graph $G=(V, E)$ of order atleast two, with order $p$ and size $q$ is called vertex-graceful if there exists a bijection $f: V \rightarrow$ $\{1,2,3, \cdots p\}$ such that the induced function $f^{*}: E \rightarrow\{0,1,2, \cdots q-$ $1\}$ defined by $f^{*}(u v)=(f(u)+f(v))(\bmod q)$ is a bijection. The bijection $f$ is called a vertex-graceful labeling of $G$. A subset $S$ of the set of natural numbers $N$ is called consecutive if $S$ consists of consecutive integers. For any set $X$, a mapping $f: X \rightarrow N$ is said to be consecutive if $f(X)$ is consecutive. A vertex-graceful labeling $f$ is said to be strong if the function $f_{1}: E \rightarrow N$ defined by $f_{1}(e)=f(u)+$ $f(v)$ for all edges $e=u v$ in $E$ forms a consecutive set. It is proved that one vertex union of odd number of copies of isomorphic caterpillars is vertex-graceful and any caterpillar is strong vertex-graceful. It is proved that a spider with even number of legs (paths) of equal length appended to each vertex of an odd cycle is vertex-graceful. It is also proved that the graph $l A\left(m_{j}, n\right)$ is vertex-graceful for both $n$ and $l$ odd, $0 \leq i \leq n-1,1 \leq j \leq m_{i}$. Further, it is proved that the graph $A\left(m_{j}, n\right)$ is strong vertex-graceful for $n$ odd, $0 \leq i \leq n-1,1 \leq j \leq$ $m_{i}$.
\end{abstract}

Key words: Caterpillar, one vertex union graphs, regular spider, actinia graph, vertex-graceful labeling, strong vertex-graceful labeling.

2010 Mathematics Subject Classification: 05C78. 


\section{Introduction}

By a graph $G=(V, E)$, we mean a finite undirected graph without loops or multiple edges. The order and size of $G$ are denoted by $p$ and $q$, respectively. For basic graph theoretic terminology, we refer to $[5,8]$. A caterpillar is a tree, the removal of whose end vertices, gives a path. The resulting path is called a spine. Denote by $C\left(n, m_{i}\right)$, a caterpillar, where $n$ is the number of vertices on the spine and $m_{i}$ is the number of end vertices adjacent to each vertex of the spine. Let $G_{i}(1 \leq i \leq l)$ be a connected graph with vertex set $V_{i}=\left\{v_{1}^{i}, v_{2}^{i}, v_{3}^{i}, \cdots, v_{n_{l}}^{i}\right\}$. Then the one vertex union of $G_{i}(1 \leq i \leq l)$ at $v_{1}^{1}$ is obtained by identifying all the vertices $v_{1}^{1}, v_{1}^{2}, v_{1}^{3}, \cdots, v_{1}^{l}$ at $v_{1}^{1}$ and is denoted by $G^{(l)}$.

A spider is a tree that has at most one vertex (called the center) of degree greater than 2. A regular spider is the one vertex union of paths of equal length. A bamboo tree is a rooted tree consisting of branches of equal length, the end points of which are identified with the end points of stars of different sizes. A bamboo tree is called regular if the sizes of the stars considered are equal. The bamboo trees were introduced and studied in [11]. The graph $A\left(m_{i}, n\right)$ obtained by identifying $m_{i},(1 \leq i \leq n)$ pendant edges to the vertices of a cycle $C_{n}$ is called Actinia graph. The Actinia graphs were introduced and studied in [19].

A graph labeling is an assignment of numbers to the vertices or edges, or both vertices and edges subject to certain conditions. A graph $G$ is called graceful graph if there exists an injection $f: V \rightarrow\{0,1,2, \cdots, q\}$ such that the induced function $f^{*}: E \rightarrow\{1,2, \cdots, q\}$ defined by $f^{*}(u v)=$ $|f(u)-f(v)|$ is a bijection. The injection $f$ is called a graceful labeling of graph $G$. The values $f(u)$ and $f^{*}(u v)$ are called thegraceful labels of the vertex $u$ and the edge $u v$, respectively. A graph $G$ is called vertex-graceful if there exists a bijection $f: V \rightarrow\{0,1,2, \cdots, p\}$ such that the induced function $f^{*}: E \rightarrow\{0,1,2, \cdots, q-1\}$ by $f^{*}(u v)=(f(u)+f(v))(\bmod q)$ is a bijection. The bijection $f$ is called a vertex-graceful labeling of $G$ - Let $N$ denote the set of natural numbers. A subset $S$ of $N$ is called consecutive if $S$ consists of consecutive integers. For any set $X$, a mapping $f: X \rightarrow N$ is said to be consecutive if $f(X)$ is consecutive. A vertexgraceful labeling $f$ is said to be strong if the function by $f_{1}: E \rightarrow N$ defined by $f_{1}(e)=f(u)+f(v)$ for all edges $e=u v$ in $E$ forms a consecutive set.

In 1967, Rosa [12] introduced the labeling method called $\beta$-valuation. A function $f$ is called $\beta$-valuation of a graph $G$ with $q$ edges if $f$ is an injection from the vertices of $G$ to the set $\{0,1,2, \cdots, q\}$ such that, when 
each edge $x y$ is assigned the label $|f(x)-f(y)|$, the resulting edge labels are distinct. Golomb [7] called such labelings graceful and this term is followed presently. The book edited by Acharia, Arumugam and Rosa [1] includes a variety of labeling methods. Hsu and Keedwell $[9,10]$ introduced and studied the extension of graceful labeling of directed graphs. The relationship between graceful directed graphs and a variety of algebraic structures including cyclic difference sets, sequenceable groups, generalized complete mappings, near-complete mapping and neofield is discussed in [3] and [4]. Bahl, Lake and Wertheim [2] proved that spiders for which the lengths of every path from the center to a leaf differ by at most one are graceful and spiders for which the lengths of every path from the center to a leaf has the same length and there is an odd number of such paths there is a family of graceful labelings.

Graph labeling, where the vertices are assigned values subject to certain conditions have often been motivated by practical problems. Labeled graphs serve as useful mathematical models for a broad range of applications such as coding theory, including the design of good radar type codes, synch-set codes, missile guidance codes and convolution codes with optimal autocorrelation properties. The notion of vertex-graceful graph of order $p$ and size $p+1$ was introduced and studied in [18]. The vertex graceful labeling of many classes of graphs were studied in [13, 14, 15, 16, 17]. For detailed study of labeling of graphs, we refer to [6].

\section{Vertex Gracefulness of One Vertex Union of Caterpillars}

In this section, it is proved that one vertex union of odd number of copies of isomorphic caterpillars is vertex-graceful and also proved that any caterpillar is strong vertex graceful.

Let $G=C^{(l)}\left(n, m_{i}\right)$ be the one vertex union of $l$ copies of isomorphic caterpillars with $n$ vertices in the spine and each of its vertices are appended with $m_{i}(1 \leq i \leq n)$ pendant vertices. Let $v_{1}^{k}, v_{2}^{k}, v_{3}^{k}, \cdots, v_{n}^{k}$ be the vertices of the spine of the $k^{\text {th }}$ copy of the isomorphic caterpillars. Let $m_{1}, m_{2}, m_{3}, \cdots, m_{n}$ be the number of pendant vertices, which are appended to each vertex of the spine of the caterpillar. Let $v_{1}^{1}, v_{1}^{2}, v_{1}^{3}, \cdots, v_{1}^{l}$ be the initial vertex of each spine of $l$ isomorphic caterpillars. The one vertex union of $l$ copies of isomorphic caterpillars at $v_{1}^{1}$ is obtained by identifying all the vertices $v_{1}^{1}, v_{1}^{2}, v_{1}^{3}, \cdots, v_{1}^{l}$ at $v_{1}^{1}$. Let $v_{i j}^{k}$ be the pendant vertices of $G$ 
for $1 \leq i \leq n, 1 \leq j \leq m_{i}, 1 \leq k \leq l$. This graph is shown in Figure 2.1.

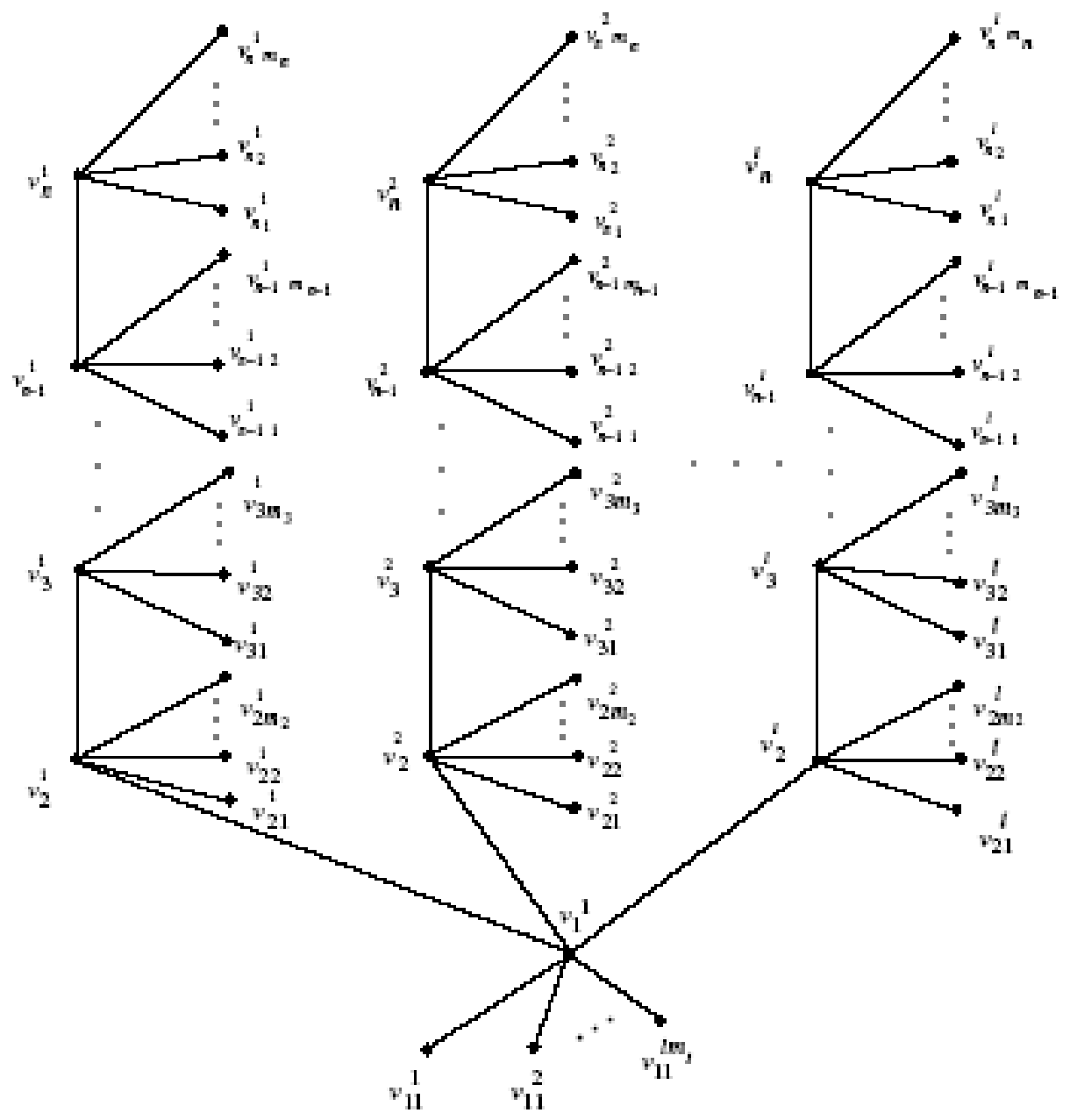

Figure 2.1: One vertex union of $l$ copies of isomorphic caterpillars

Theorem 2.1. For $1 \leq i \leq n, 1 \leq j \leq m_{i}$ and $l$ odd, the graph $G=$ $C^{(l)}\left(n, m_{i}\right)$ is vertex-graceful. 
Proof. It is clear from the definition of $G$ that the number of vertices $p$ and edges $q$ are $p=l\left(n-1+\sum_{a=1}^{n} m_{a}\right)+1$ and $q=p-1$. Let $\alpha=n-1+\sum_{a=1}^{n-1} m_{a+1}$. The required vertex-graceful labeling for vertices is defined as follows:

Let $f: V \rightarrow\{1,2, \cdots, p\}$.

Case 1. $n$ even.

Let $\beta=\sum_{a=1}^{\frac{n-2}{2}} m_{2 a}$

Define $f\left(v_{1}^{1}\right)=1$,

$$
\begin{aligned}
& \mathrm{f}\left(\mathrm{v}_{i}^{k}\right)=\left\{\alpha(k-1) 2+\frac{i+1}{2}+\sum_{a=1}^{\frac{i-1}{2}} m_{2 a}, \quad 3 \leq i \leq n, i \text { odd } 1 \leq k \leq l, k\right. \text { odd. } \\
& \frac{\alpha(l+k-2)}{2}+\beta+l m_{1}+m_{n}+\frac{n+i}{2}+\sum_{a=1}^{\frac{i-2}{2}} m_{2 a+1}, \quad 2 \leq i \leq n, i \text { even; } \\
& \frac{\alpha(k-2)}{2}+\beta+m_{n}+\frac{n+i}{2}+\sum_{a=1}^{\frac{i-2}{2}} m_{2 a+1}, \quad 2 \leq i \leq n, i \text { even; } 1 \leq k \leq l, k \text { even. } \\
& \frac{\alpha(l+k-1)}{2}+l m_{1}+\frac{i+1}{2}+\sum_{a=1}^{\frac{i-1}{2}} m_{2 a}, \quad 2 \leq i \leq n, i \text { odd } 1 \leq k \leq l, k \text { even. }
\end{aligned}
$$

The vertex labeling of pendant vertices at $v_{1}^{k}, v_{2}^{k}, v_{3}^{k}, \cdots, v_{n}^{k}$ is given by

$$
\begin{gathered}
\mathrm{f}\left(\mathrm{v}_{i j}^{k}\right)=\left\{\alpha(l-1) 2+\beta+\frac{n}{2}+m_{n}+k, \text { for } i=j=1 ; 1 \leq k \leq l m_{1} .\right. \\
\frac{\alpha(l+k-2)}{2}+\beta+l m_{1}+m_{n}+\frac{n+i-1}{2}+j+\sum_{a=1}^{\frac{i-3}{2}} m_{2 a+1},
\end{gathered}
$$

$2 \leq i \leq n, i$ odd $; \leq j \leq m_{i} ; 1 \leq k \leq l, k$ odd.

$$
\frac{\alpha(k-1)}{2}+\frac{i}{2}+j+\sum_{a=1}^{\frac{i-2}{2}} m_{2 a}
$$

$2 \leq i \leq n, i$ even $; 1 \leq j \leq m_{i} ; 1 \leq k \leq l, k$ odd.

$\frac{\alpha(k-2)}{2}+\beta+m_{n}+\frac{n+i-1}{2}+j+\sum_{a=1}^{\frac{i-3}{2}} m_{2 a+1}$,

$2 \leq i \leq n, i$ odd $; 1 \leq j \leq m_{i} ; 1 \leq k \leq l, k$ even. 
$\frac{\alpha(l+k-1)}{2}+l m_{1}+\frac{i}{2}+j+\sum_{a=1}^{\frac{i-2}{2}} m_{2 a}$

$2 \leq i \leq n, i$ even $1 \leq j \leq m_{i} ; 1 \leq k \leq l, k$ even.

The corresponding edge labeling is defined as follows:

Let $A=\left\{f^{*}\left(e_{11}^{k}\right): f^{*}\left(e_{11}^{k}\right)=\left(\frac{\alpha(l-1)}{2}+\frac{n}{2}+m_{n}+k+1+\beta\right)(\bmod q)\right\}$, where $e_{11}^{k}=v_{1}^{1} v_{11}^{k}, 1 \leq k \leq l m_{1}$.

Let $\delta=\frac{n}{2}+l m_{1}+m_{n}+\beta$,

$B=\left\{f^{*}\left(e_{i}^{k}\right): f^{*}\left(e_{i}^{k}\right)=\left(\frac{\alpha(l+2 k-3)}{2}+1+\delta+i+\sum_{a=1}^{i-1} m_{a+1}\right)(\bmod q)\right\}$,

where $e_{i}^{k}=v_{i}^{k} v_{i+1}^{k}, 2 \leq i \leq n ; 1 \leq k \leq l$;

$C=\left\{f^{*}\left(e_{i j}^{k}\right): f^{*}\left(e_{i j}^{k}\right)=\left(\frac{\alpha(l+2 k-3)}{2}+\delta+i+j+\sum_{a=1}^{i-2} m_{a+1}\right)(\bmod q)\right\}$ where $e_{i j}^{k}=v_{i}^{k} v_{i j}^{k}, 2 \leq i \leq n ; 1 \leq j \leq m_{i} ; 1 \leq$

$k \leq l$

$$
\begin{gathered}
D=\left\{f^{*}\left(e_{1}^{k}\right): f^{*}\left(e_{1}^{k}\right)=\left(\frac{\alpha(l+k-2)}{2}+\delta+2\right)(\bmod q)\right\}, \\
\quad \text { where } e_{1}^{k}=v_{1}^{1} v_{2}^{k}, 1 \leq k \leq l, k \text { odd } ; \\
E=\left\{f^{*}\left(e_{1}^{k}\right): f^{*}\left(e_{1}^{k}\right)=\left(\begin{array}{c}
\left.\left.\frac{\alpha(k-2)}{2}+\beta+m_{n}+\frac{n+4}{2}\right)(\bmod q)\right\} \\
\quad \text { where } e_{1}^{k}=v_{1}^{1} v_{2}^{k}, 1 \leq k \leq l, k \text { even } .
\end{array}\right.\right.
\end{gathered}
$$

It is clear that all the vertex labels are distinct. Also it is easily verified that the edge label sets $A, B, C, D$ and $E$ are mutually disjoint and $A \cup B \cup C \cup D \cup E=\{0,1,2, \cdots, q-1\}$. Therefore, it follows that the induced mapping $f^{*}: E \rightarrow\{0,1,2, \cdots, q-1\}$ is a bijection. Hence the graph $G$ is a vertex-graceful when $n$ is even.

Case 2. $n$ odd.

Let $\gamma=\sum_{a=1}^{\frac{n-1}{2}} m_{2 a}$ and $\mu=\gamma+l m_{1}+\frac{n+1}{2}$.

Define $f\left(v_{1}^{1}\right)=1$, 


$$
\begin{aligned}
& \mathrm{f}\left(\mathrm{v}_{i}^{k}\right)=\left\{\alpha(k-1) 2+\frac{i+1}{2}+\sum_{a=1}^{\frac{i-1}{2}} m_{2 a}, \quad 2 \leq i \leq n, i \text { odd } 1 \leq k \leq l, k\right. \text { odd. } \\
& \frac{\alpha(l+k-2)}{2}+\gamma+l m_{1}+\frac{n+i+1}{2}+\sum_{a=1}^{\frac{i-2}{2}} m_{2 a+1}, \quad 2 \leq i \leq n, i \text { even; } \\
& \frac{\alpha(k-2)}{2}+\gamma+\frac{n+i+1}{2}+\sum_{a=1}^{\frac{i-2}{2}} m_{2 a+1}, \quad 2 \leq i \leq n, i \text { even; } 1 \leq k \leq l, k \text { even. } \\
& \frac{\alpha(l+k-1)}{2}+l m_{1}+\frac{i+1}{2}+\sum_{a=1}^{\frac{i-1}{2}} m_{2 a}, \quad 2 \leq i \leq n, i \text { odd } 1 \leq k \leq l, k \text { even. }
\end{aligned}
$$

The vertex labeling of pendant vertices at $v_{1}^{k}, v_{2}^{k}, v_{3}^{k}, \cdots, v_{n}^{k}$ is given by $\mathrm{f}\left(\mathrm{v}_{i j}^{k}\right)=\left\{\alpha(l-1) 2+\frac{n+1}{2}+k+\gamma\right.$, for $i=j=1 ; 1 \leq k \leq l m_{1}$.

$\frac{\alpha(k-1)}{2}+\frac{i}{2}+j+\sum_{a=1}^{\frac{i-2}{2}} m_{2 a}$,

$2 \leq i \leq n, i$ even; $1 \leq j \leq m_{i} ; 1 \leq k \leq l, k$ odd.

$\frac{\alpha(l+k-2)}{2}+\gamma+l m_{1}+\frac{n+i}{2}+j+\sum_{a=1}^{\frac{i-3}{2}} m_{2 a+1}$,

$2 \leq i \leq n, i$ odd $; 1 \leq j \leq m_{i} ; 1 \leq k \leq l, k$ odd.

$\frac{\alpha(k-2)}{2}+\gamma+\frac{n+i}{2}+j+\sum_{a=1}^{\frac{i-3}{2}} m_{2 a+1}$,

$2 \leq i \leq n, i$ odd $; 1 \leq j \leq m_{i} ; 1 \leq k \leq l, k$ even.

$\frac{\alpha(l+k-1)}{2}+l m_{1}+\frac{i}{2}+j+\sum_{a=1}^{\frac{i-2}{2}} m_{2 a}$

$2 \leq i \leq n, \quad i$ even; $1 \leq j \leq m_{i} ; 1 \leq k \leq l, k$ even.

The corresponding edge labeling is defined as follows:

Let $A=\left\{f^{*}\left(e_{1}^{k}\right): f^{*}\left(e_{1}^{k}\right)=\left(\frac{\alpha(l+k-2)}{2}+\gamma+l m_{1}+\frac{n+5}{2}\right)(\bmod q)\right\}$, where $e_{1}^{k}=v_{1}^{1} v_{2}^{k}, 1 \leq k \leq l, k$ odd ;

$$
\begin{aligned}
& B=\left\{f^{*}\left(e_{1}^{k}\right): f^{*}\left(e_{1}^{k}\right)=\left(\frac{\alpha(k-2)}{2}+\gamma+\frac{n+5}{2}\right)(\bmod q)\right\}, \\
& \text { where } e_{1}^{k}=v_{1}^{k} v_{2}^{k}, 1 \leq k \leq l, k \text { even; } \\
& C=\left\{f^{*}\left(e_{i}^{k}\right): f^{*}\left(e_{i}^{k}\right)=\left(\frac{\alpha(l+2 k-3)}{2}+i+1+\mu+\sum_{a=1}^{i-1} m_{a+1}\right)(\bmod q)\right\}, \\
& \text { where } e_{i}^{k}=v_{i}^{k} v_{i+1}^{k}, 2 \leq i \leq n-1 ; 1 \leq k \leq l \text {; }
\end{aligned}
$$




$$
D=\left\{f^{*}\left(e_{i j}^{k}\right): f^{*}\left(e_{i j}^{k}\right)=\left(\frac{\alpha(l+2 k-3)}{2}+i+j+\mu+\sum_{a=1}^{i-2} m_{a+1}\right)(\bmod q)\right\}
$$

$l$; where $e_{i j}^{k}=v_{i}^{k} v_{i j}^{k}, 2 \leq i \leq n ; 1 \leq j \leq m_{i} ; 1 \leq k \leq$

$$
\begin{aligned}
& E=\left\{f^{*}\left(e_{11}^{k}\right): f^{*}\left(e_{11}^{k}\right)=\left(\frac{\alpha(l-1)}{2}+\frac{n+1}{2}+k+\gamma+1\right)(\bmod q)\right\}, \\
& \text { where } e_{11}^{k}=v_{1}^{k} v_{11}^{k}, 1 \leq k \leq l m_{1} \text {. }
\end{aligned}
$$

It is clear that all the vertex labels are distinct. Also it is easily verified that the edge label sets $A, B, C, D$ and $E$ are mutually disjoint and $A \cup B \cup C \cup D \cup E=\{0,1,2, \cdots, q-1\}$. Therefore, it follows that the induced mapping $f^{*}: E \rightarrow\{0,1,2, \cdots, q-1\}$ is a bijection. Hence the graph $G$ is a vertex-graceful when $n$ is odd. Thus the graph $G$ is vertex-graceful.

Illustrative examples of the labeling of the graph $G$ in Theorm 2.1 are given in Figures 2.2 and 2.3.

We leave the following problem as an open question.

Problem 2.2. Is it true that for $1 \leq i \leq n, 1 \leq j \leq m_{i}$ and $l$ even, the graph $G=C^{(l)}\left(n, m_{i}\right)$ is vertex-graceful? 


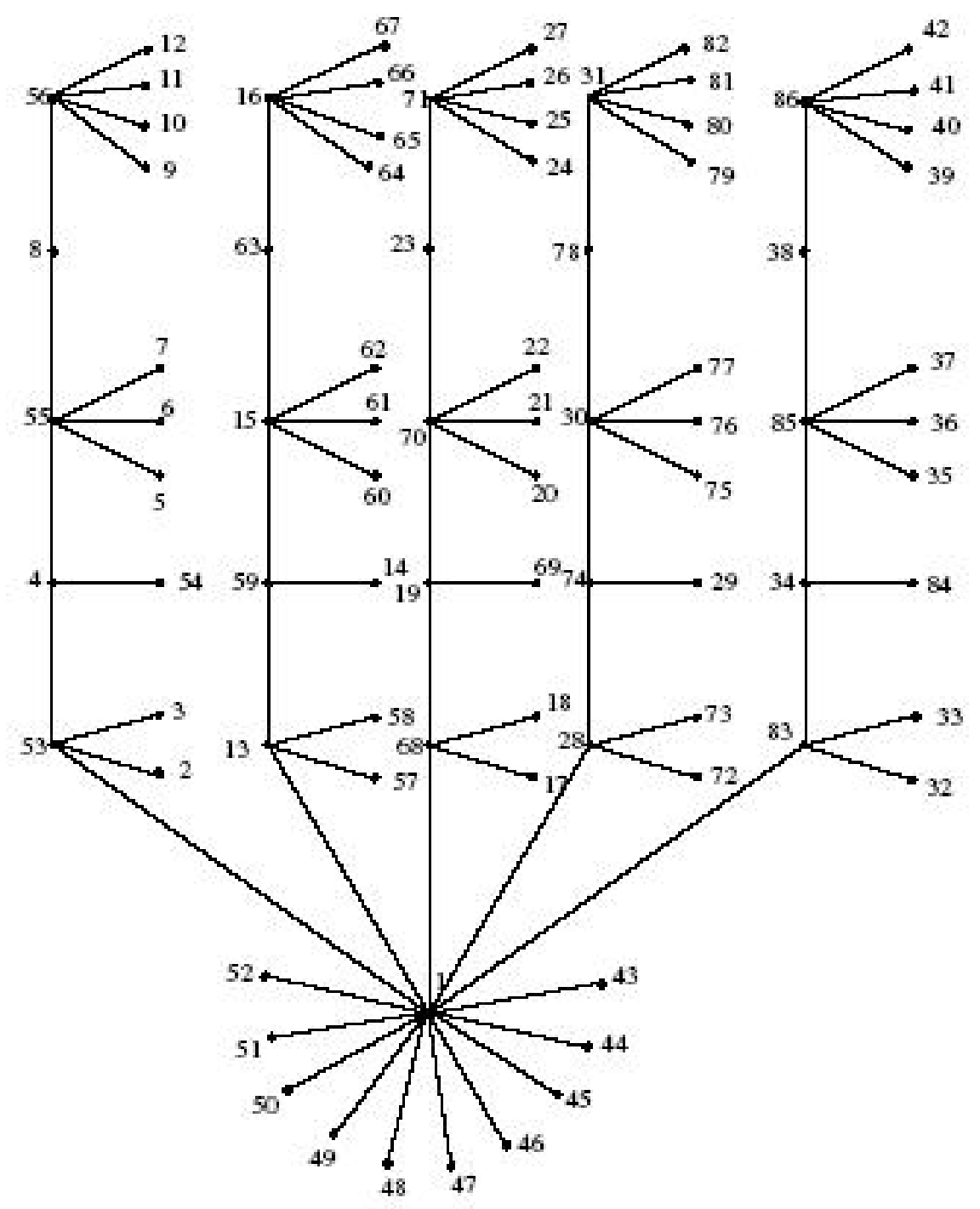

Figure 2.2: Vertex-graceful labeled graph of $C^{5}\left(6, m_{1}\right), 1 \leq i \leq 6, m_{1}=$ $2, m_{2}=2, m_{3}=1, m_{4}=3, m_{5}=0, m_{6}=4$ (case 1 ) 


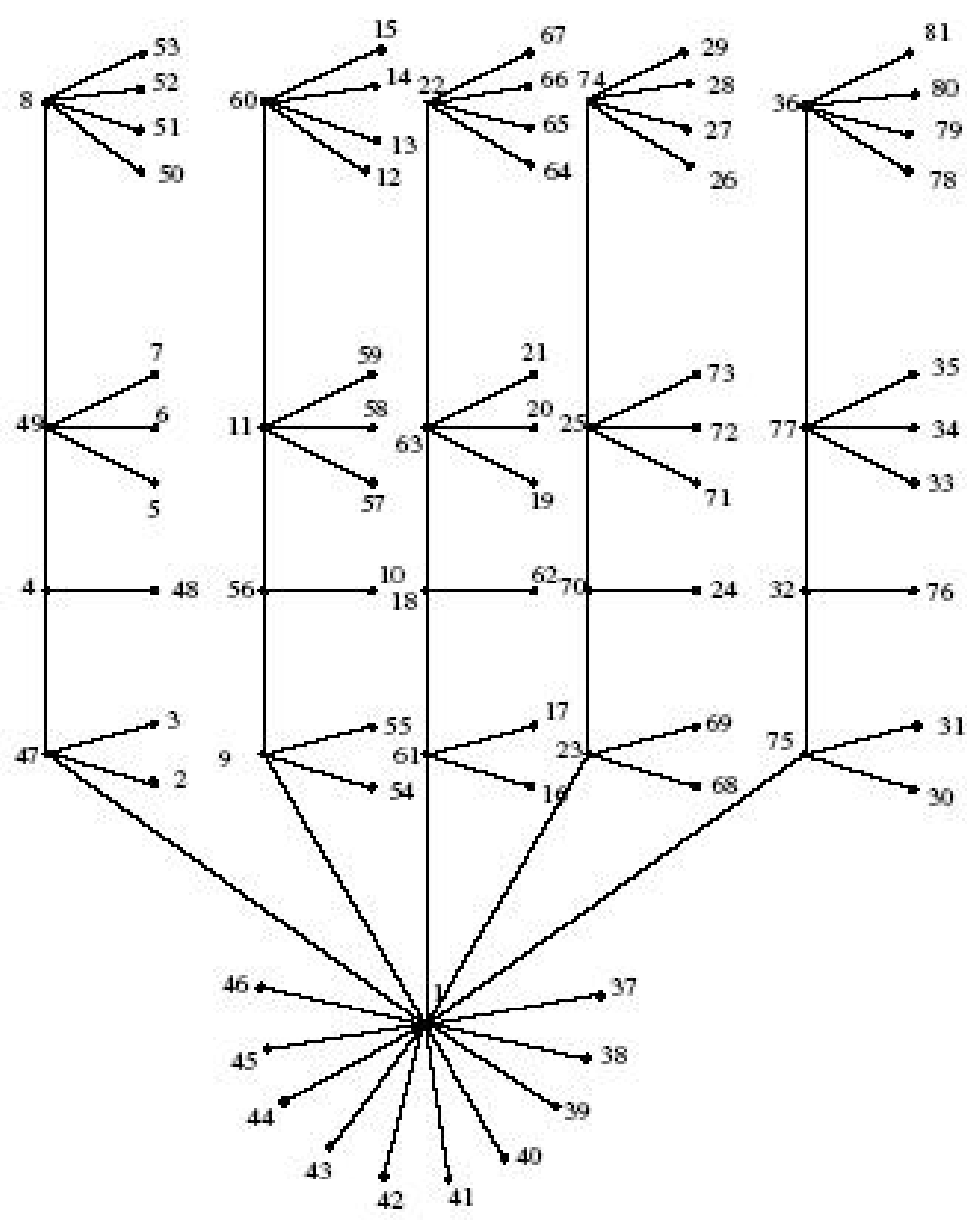

Figure 2.3: Vertex-graceful labeled graph of $C^{5}\left(5, m_{i}\right), m_{1}=2, m_{2}=2, m_{3}=$ $1, m_{4}=3, m_{5}=4$ (case 2 )

Remark 2.3. If $l=1$ in Figure 1 , then the graph $G$ represents a caterpillar. Hence it follows from Theorem 2.1 that any caterpillar is vertexgraceful. Also if $m_{i}=0$ for $i=1,2, \cdots, n-1$ and $m_{n} \neq 0$ in Figure 2.1, then the graph $G$ represents a regular bamboo tree. Hence it follows from Theorem 2.1 that a regular bamboo tree is vertex-graceful.

Theorem 2.4. Any caterpillar $G$ is strong vertex graceful. 
Proof. Let the caterpillar $G$ be a tree with $n$ vertices $v_{1}, v_{2}, \cdots, v_{n}$ on the spine and each $v_{i}$ is attached with $m_{i}$ pendant edges $(1 \leq i \leq n)$. The graph is given in Figure 2.4.

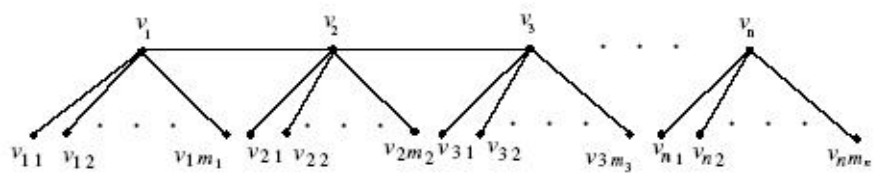

Figure 2.4: G

Then $p=n+\sum_{a=1}^{n} m_{a}$ and $q=n-1+\sum_{a=1}^{n} m_{a}$. The required strong vertex-graceful labeling for vertices is defined as follows:

Let $f: V \rightarrow\{1,2, \cdots, p\}$ be such that

Case 1. $n$ even.

Let $\beta=\sum_{a=1}^{\frac{n-2}{2}} m_{2 a}$

$$
\begin{aligned}
& \mathrm{f}\left(\mathrm{v}_{i}\right)=\left\{i+12+\sum_{a=1}^{\frac{i-1}{2}} m_{2 a}, 1 \leq i \leq n, i\right. \text { odd. } \\
& \beta+m_{1}+m_{n}+\frac{n+i}{2}+\sum_{a=1}^{\frac{i-2}{2}} m_{2 a+1}, 1 \leq i \leq n, i \text { even. }
\end{aligned}
$$

The vertex labeling of pendant vertices at $v_{1}, v_{2}, v_{3}, \cdots, v_{n}$ is given by $\mathrm{f}\left(\mathrm{v}_{i j}\right)=\left\{\beta+m_{n}+\frac{n+i-1}{2}+j+\sum_{a=1}^{\frac{i-1}{2}} m_{2 a-1}, 1 \leq i \leq n, i\right.$ odd $1 \leq j \leq m_{i}$. $\frac{i}{2}+j+\sum_{a=1}^{\frac{i-2}{2}} m_{2 a}, 1 \leq i \leq n, \quad i$ even $1 \leq j \leq m_{i}$.

The corresponding edge labeling is defined as follows:

Let $A=\left\{f_{1}\left(e_{i j}\right): f_{1}\left(e_{i j}\right)=\left(\frac{n}{2}+m_{n}+\beta+i+j+\sum_{a=1}^{i-1} m_{a}\right)\right\}$,

where $e_{i j}=v_{i} v_{i j}, 1 \leq i \leq n ; 1 \leq j \leq m_{i}$. 


$$
B=\left\{f_{1}\left(e_{i}\right): f_{1}\left(e_{i}\right)=\left(\begin{array}{r}
\left.\left.\frac{n}{2}+m_{n}+\beta+1+i+\sum_{a=1}^{i} m_{a}\right)\right\} \\
\text { where } e_{i}=v_{i} v_{i+1}, 1 \leq i \leq n-1 .
\end{array}\right.\right.
$$

It is clear that the vertex labels are distinct. Also it is easily verified that the edge labels sets are mutually disjoint and $A \cup B=\left\{\frac{n}{2}+\beta+m_{n}+\right.$ $\left.2, \frac{n}{2}+\beta+m_{n}+3, \cdots, \frac{n}{2}+\beta+m_{n}+p\right\}$. Therefore, the induced edge labels of $G$ have $q$ consecutive values. Hence the graph $G$ is strong vertex-graceful when $n$ is even.

Case 2. $n$ odd.

Let $\gamma=\sum_{a=1}^{\frac{n-1}{2}} m_{2 a}$

$\mathrm{f}\left(\mathrm{v}_{i}\right)=\left\{i+12+\sum_{a=1}^{\frac{i-1}{2}} m_{2 a}, 1 \leq i \leq n, i\right.$ odd.
$\gamma+m_{1}+\frac{n+i+1}{2}+\sum_{a=1}^{\frac{i-2}{2}} m_{2 a+1}, 1 \leq i \leq n, i$ even.

The vertex labeling of pendant vertices at $v_{1}, v_{2}, v_{3}, \cdots, v_{n}$ is given by $\mathrm{f}\left(\mathrm{v}_{i j}\right)=\left\{\gamma+\frac{n+i}{2}+j+\sum_{a=1}^{\frac{i-1}{2}} m_{2 a-1}, 1 \leq i \leq n, i\right.$ odd $1 \leq j \leq m_{i}$. $\frac{i}{2}+j+\sum_{a=1}^{\frac{i-2}{2}} m_{2 a}, 1 \leq i \leq n, i$ even $1 \leq j \leq m_{i}$.

The corresponding edge labeling is defined as follows: Let $A=\left\{f_{1}\left(e_{i j}\right): f_{1}\left(e_{i j}\right)=\left(\frac{n+1}{2}+\gamma+i+j+\sum_{a=1}^{i-1} m_{a}\right)\right\}$, where $e_{i j}=v_{i} v_{i j}, 1 \leq i \leq n ; 1 \leq j \leq m_{i}$.

$$
B=\left\{f_{1}\left(e_{i}\right): f_{1}\left(e_{i}\right)=\left(\frac{n+1}{2}+\gamma+1+i+\sum_{a=1}^{i} m_{a}\right)\right\}
$$
where $e_{i}=v_{i} v_{i+1}, 1 \leq i \leq n-1$.

It is clear that the vertex labels are distinct. Also it is easily verified that the edge labels sets are mutually disjoint and $A \cup B=\left\{\frac{n+1}{2}+\gamma+\right.$ $\left.2, \frac{n+1}{2}+\gamma+3, \cdots, \frac{n+1}{2}+\gamma+p\right\}$. Therefore the induced edge labels of $G$ have $q$ consecutive values. Hence the graph $G$ is strong vertex-graceful when $n$ 
is odd. Thus the graph $G$ is strong- vertex graceful.

\section{Vertex-graceful Labeling of Cycle with Regular Spider}

In this section, it is proved that a spider with even number of legs (paths) of equal length appended to each vertex of an odd cycle is vertexgraceful.

We start with the following definitions :

Definition 3.1. A subdivision of an edge $u v$ in a graph is obtained by removing edge $u v$, adding a new vertex $w$ and adding edges $u w$ and $v w$. A (wounded) spider is the graph formed by subdividing at most $t-1$ of the edges of a star $K_{1, t}$ for $t \geq 0$.

Examples of wounded spiders include $K_{1}$, the star $K_{1, n-1}$, and the graph shown in Figure 3.1.

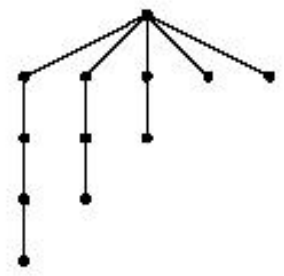

Figure 3.1: A wounded spider

Definition 3.2. A regular spider is the one vertex union of paths of equal length. Denote by $C_{n} P_{n}^{(l)}$ a cycle $C_{m}(m \geq 3)$ attached at each of its vertices a regular spider $P_{n}^{(l)}$, where $l$ denotes the number of paths $P_{n}$ in the spider (Refer Figure 3.2).

\section{Example 3.3.}




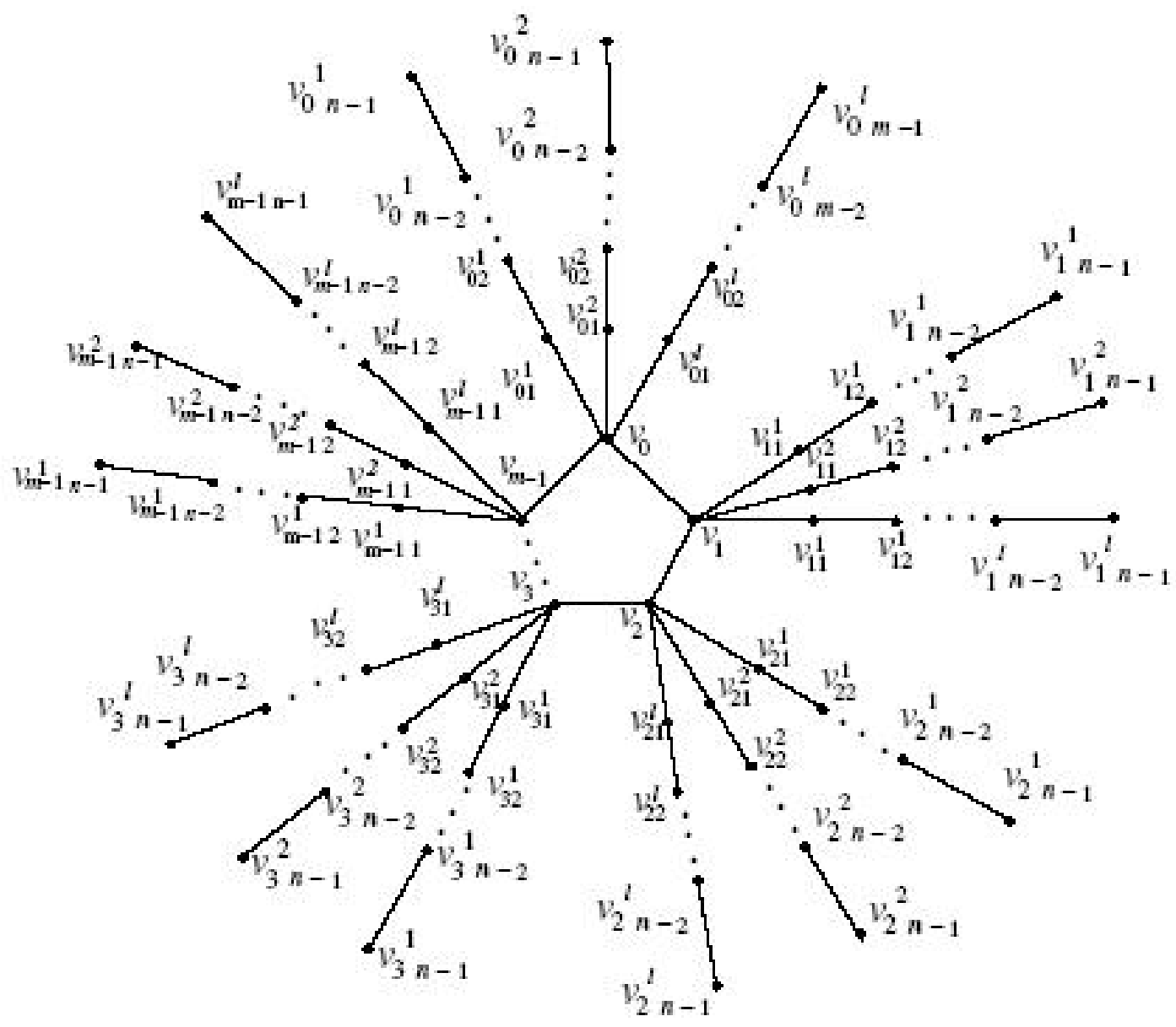

Figure 3.2: The graph $C_{n} \cdot P_{n}^{(l)}$

Theorem 3.4. For any $n$, the graph $C_{n} P_{n}^{(l)}$ is vertex-graceful where $m$ odd and $l$ even. 
Proof. Let $v_{0}, v_{1}, \cdots, v_{m-1}$ be the vertices of the cycle $C_{m}$. Now, let $C_{n} P_{n}^{(l)}$ be the graph as shown in Figure 6 . It is easily verified that $G$ has $p=m[l(n-1)+1]$ vertices and $q=p$ edges. Let $\alpha=l(n-1)+1$. The required vertex-graceful labeling for vertices is defined as follows :

Let $f: V \rightarrow\{1,2, \cdots, p\}$ be such that

Case 1. $n$ even.

Define $f\left(v_{i}\right)=\alpha i+1,0 \leq i \leq m-1$,

$\mathrm{f}\left(\mathrm{v}_{i j}^{k}\right)=\left\{\alpha i+\frac{(n-1)(k-1)}{2}+\frac{j+2}{2}\right.$,

$0 \leq i \leq m-1 ; 1 \leq j \leq n-1, j$ even; $1 \leq k \leq l, k$ odd.

$\alpha i+\frac{(n-1)(k-1)}{2}+\beta+\frac{j+3}{2}$,

$0 \leq i \leq m-1 ; 1 \leq j \leq n-1, j$ odd $; 1 \leq k \leq l, k$ odd.

$\alpha i+\frac{k(n-1)}{2}+\frac{3-j}{2}$,

$0 \leq i \leq m-1 ; 1 \leq j \leq n-1, j$ odd; $1 \leq k \leq l, k$ even.

$\alpha i+\beta+\frac{k(n-1)}{2}+2-\frac{j}{2}$,

$0 \leq i \leq m-1 ; 1 \leq j \leq n-1, j$ even; $1 \leq k \leq l, k$ even.

The corresponding edge labeling is defined as follows:

Let $A=\left\{f^{*}\left(e_{i}\right): f^{*}\left(e_{i}\right)=(\alpha(2 i+1)+2)(\bmod q)\right\}$, where $e_{i}=v_{i} v_{(i+1) \bmod m}, 0 \leq i \leq m-1$.

$$
\begin{array}{r}
B=\left\{f^{*}\left(e_{i j}^{k}\right): f^{*}\left(e_{i j}^{k}\right)=((k-1)(n-1)+\beta+2 \alpha i+(j+3))(\bmod q)\right\}, \\
\quad \text { where } e_{i j}^{k}=v_{i j}^{k} v_{i j+1}^{k}, \quad 0 \leq i \leq m-1 ; 1 \leq j \leq n-2 ; 1 \leq k \leq
\end{array}
$$
$l, k$ odd.

$$
C=\left\{f^{*}\left(e_{i j}^{k}\right): f^{*}\left(e_{i j}^{k}\right)=(2 \alpha i+\beta+k(n-1)+3-j)(\bmod q)\right\},
$$
where $e_{i j}^{k}=v_{i j}^{k} v_{i j+1}^{k}, \quad 0 \leq i \leq m-1 ; 1 \leq j \leq n-2 ; 1 \leq k \leq$ $l, k$ even.

$$
\begin{aligned}
D= & \left\{f^{*}\left(e_{i 0}^{k}\right): f^{*}\left(e_{i 0}^{k}\right)=\left(2 \alpha i+\beta+3+\frac{(n-1)(k-1)}{2}\right)(\bmod q)\right\}, \\
& \text { where } e_{i 0}^{k}=v_{i}^{k} v_{i 1}^{k}, 0 \leq i \leq m-1 ; 1 \leq k \leq l, k \text { odd } . \\
E=\{ & \left.f^{*}\left(e_{i 0}^{k}\right): f^{*}\left(e_{i 0}^{k}\right)=\left(2 \alpha i+\frac{k(n-1)}{2}+2\right)(\bmod q)\right\} \\
& \text { where } e_{i 0}^{k}=v_{i} v_{i 1}^{k}, 0 \leq i \leq m-1 ; 1 \leq k \leq l, k \text { even. }
\end{aligned}
$$

It is clear that the vertex labels are distinct. Also it is easily verified that the edge label sets $A, B, C, D$ and $E$ are mutually disjoint and $A \cup B \cup C \cup D \cup E=\{0,1,2, \cdots, q-1\}$. Therefore, it follows that the induced mapping $f^{*}: E \rightarrow\{0,1,2, \cdots, q-1\}$ is a bijection. Hence the 
graph $G$ is vertex-graceful when $n$ is even.

Case 2. $n$ odd.

Define $f\left(v_{i}\right)=\alpha i+1,0 \leq i \leq m-1$. Let $\beta=\frac{l(n-1)}{2}$

$$
\mathrm{f}\left(\mathrm{v}_{i j}^{k}\right)=\left\{\alpha i+\frac{(k-1)}{2} \frac{(k+1)}{2} \frac{(n-1)}{2}+\frac{j+2}{2},\right.
$$

$0 \leq i \leq m-1 ; 1 \leq j \leq n-1, j$ even; $1 \leq k \leq l, k$ odd. $\alpha i+\beta+\frac{(k-1)}{2} \frac{(k+1)}{2} \frac{(n-1)}{2}+\frac{\overline{j+3}}{2}$, $0 \leq i \leq m-1 ; 1 \leq j \leq n-1, j$ odd; $1 \leq k \leq l, k$ odd
$\alpha i+\frac{k(n-1)}{2}-\frac{j-3}{2}$,
$0 \leq i \leq m-1 ; 1 \leq j \leq n-1, j$ odd; $1 \leq k \leq l, k$ even. $\alpha i+\beta+\frac{\bar{k}(n-1)}{2}+2-\frac{j}{2}$,

$0 \leq i \leq m-1 ; 1 \leq j \leq n-1, j$ even; $1 \leq k \leq l, k$ even.

The corresponding edge labeling is defined as follows:

Let $A=\left\{f^{*}\left(e_{i}\right): f^{*}\left(e_{i}\right)=(\alpha(2 i+1)+2)(\bmod q)\right\}$, where $e_{i}=v_{i} v_{(i+1) \bmod m}, 0 \leq i \leq m-1$.

$$
\begin{gathered}
B=\left\{f^{*}\left(e_{i j}^{k}\right): f^{*}\left(e_{i j}^{k}\right)=\left((k-1)\left(\frac{n-1}{2}\right)\left(\frac{k+1}{2}\right)+\beta+2 \alpha i+j+3\right)(\bmod q)\right\}, \\
\text { where } e_{i j}^{k}=v_{i j}^{k} v_{i j+1}^{k}, 0 \leq i \leq m-1 ; 1 \leq j \leq n-2 ; 1 \leq k \leq
\end{gathered}
$$
$l, k$ odd.

$$
\begin{aligned}
C= & \left\{f^{*}\left(e_{i j}^{k}\right): f^{*}\left(e_{i j}^{k}\right)=(2 \alpha i+\beta+k(n-1)+3-j)(\bmod q)\right\}, \\
& \text { where } e_{i j}^{k}=v_{i j}^{k} v_{i j+1}^{k}, 0 \leq i \leq m-1 ; 1 \leq j \leq n-2 ; 1 \leq k \leq
\end{aligned}
$$

$l, k$ even.

$$
\begin{aligned}
D= & \left\{f^{*}\left(e_{i 0}^{k}\right): f^{*}\left(e_{i 0}^{k}\right)=\left(2 \alpha i+\beta+3+\frac{(k-1)}{2} \frac{(k+1)}{2} \frac{(n-1)}{2}\right)(\bmod q)\right\}, \\
& \text { where } e_{i 0}^{k}=v_{i} v_{i 1}^{k}, 0 \leq i \leq m-1 ; 1 \leq k \leq l, k \text { odd } \\
E=\{ & \left\{f^{*}\left(e_{i 0}^{k}\right): f^{*}\left(e_{i 0}^{k}\right)=\left(2 \alpha i+\frac{k(n-1)}{2}+2\right)(\bmod q)\right\} \\
& \text { where } e_{i 0}^{k}=v_{i} v_{i 1}^{k}, 0 \leq i \leq m-1 ; 1 \leq k \leq l, k \text { even } .
\end{aligned}
$$

It is clear that the vertex labels are distinct. Also it is easily verified that the edge label sets $A, B, C, D$ and $E$ are mutually disjoint and $A \cup$ $B \cup C \cup D \cup E=\{0,1,2, \cdots, q-1\}$. Therefore, it follows that the induced mapping $f^{*}: E \rightarrow\{0,1,2, \cdots, q-1\}$ is a bijection. Hence the graph $G$ is a vertex-graceful when $n$ is odd.

Illustrative example of labeling of Theorem 3.1 is given in Figures 3.3 
and 3.4.

We leave the following problem as an open question.

Problem 3.5. For any $n$, is it true that the graph $C_{n} P_{n}^{(l)}$ is vertex-graceful when

(i) $m$ odd and $l$ odd ?

(ii) $m$ even and $l$ odd ?

(iii) $m$ even and $l$ even ?

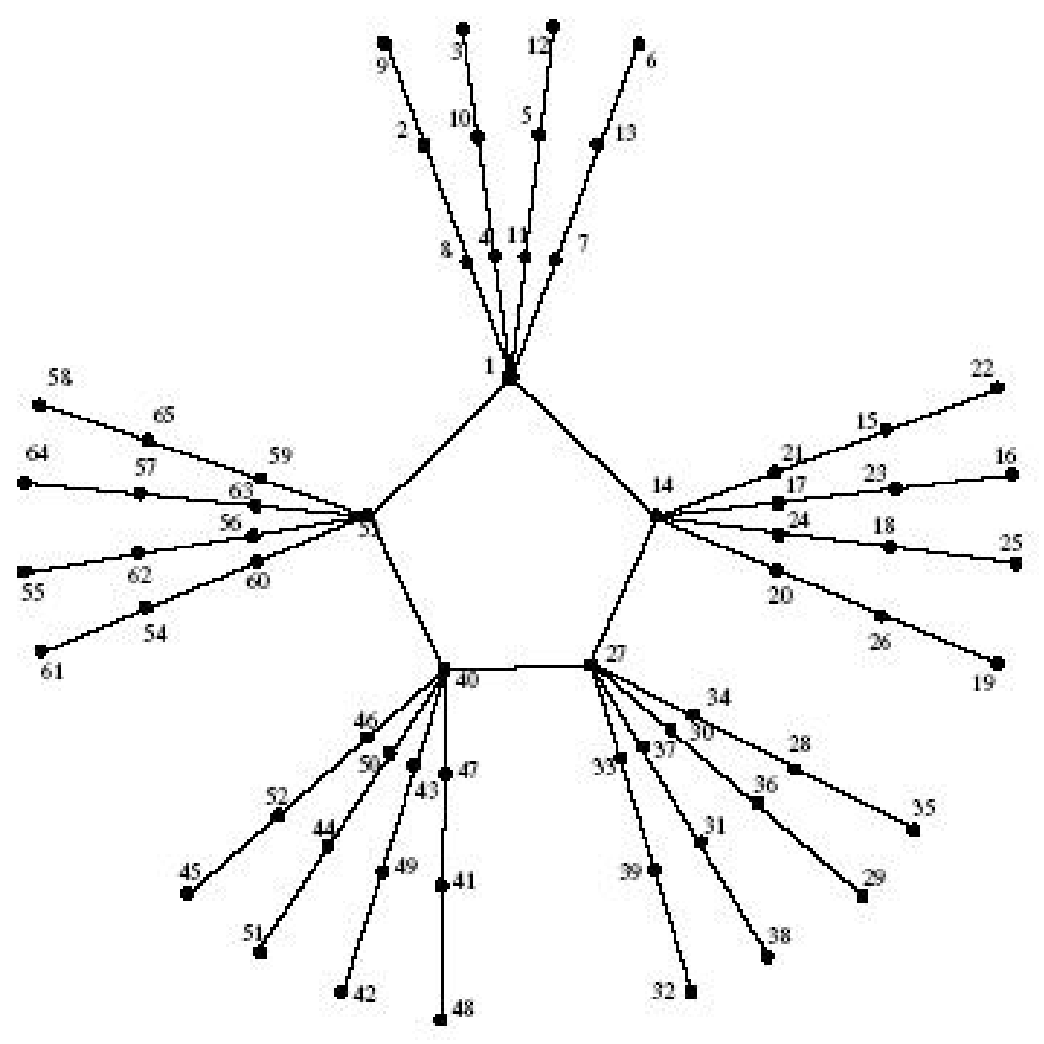

Figure 3.3: Vertex graceful labeled graph of $C_{5} \cdot P_{4}^{(4)}$ 


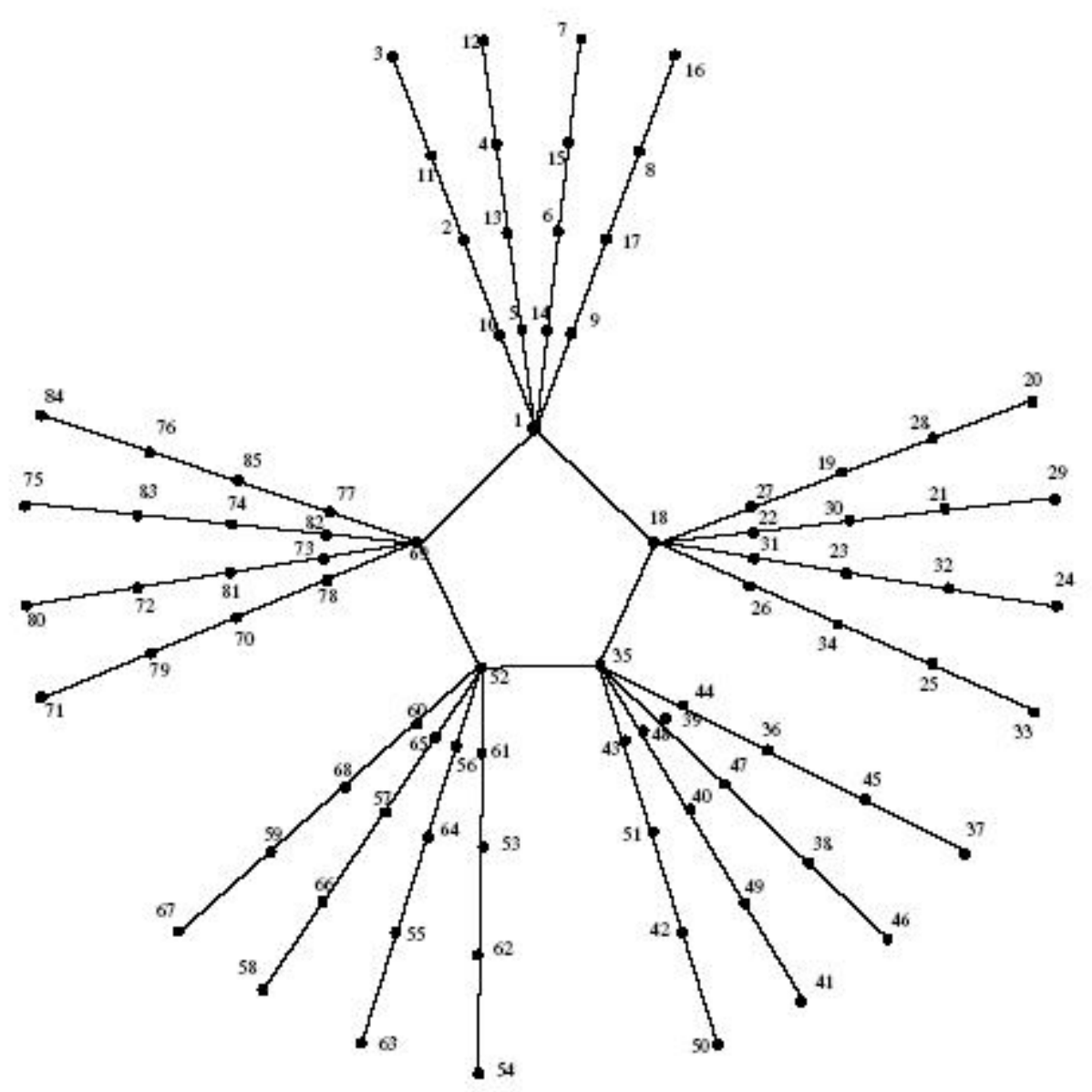

Figure 3.4: Vertex graceful labeled graph of $C_{5} \cdot P_{5}^{(4)}$

\section{Vertex-graceful Labeling of $l$ copies of Actinia Graphs}

In this section, it is proved that $l$ copies of the actinia graphs $A\left(m_{j}, n\right)$ is vertex-graceful. Let $v_{0}^{k}, v_{1}^{k}, v_{2}^{k}, \cdots, v_{n-1}^{k}$ be the vertices of $k^{\text {th }}$ copy of the cycle in $l A\left(m_{j}, n\right)$ for $0 \leq j \leq n-1$. Let $v_{i j}^{k}$ denote the $k^{t h}$ pendant vertices of the $k^{t h}$ copy of $l A\left(m_{j}, n\right)$ such that $v_{i j}^{k}$ are adjacent to vertices $v_{i}^{k}$ for $0 \leq i \leq n-1,1 \leq j \leq m_{i}, 1 \leq k \leq l$. The graph $l A\left(m_{j}, n\right)$ is shown in Figure 4.1. 

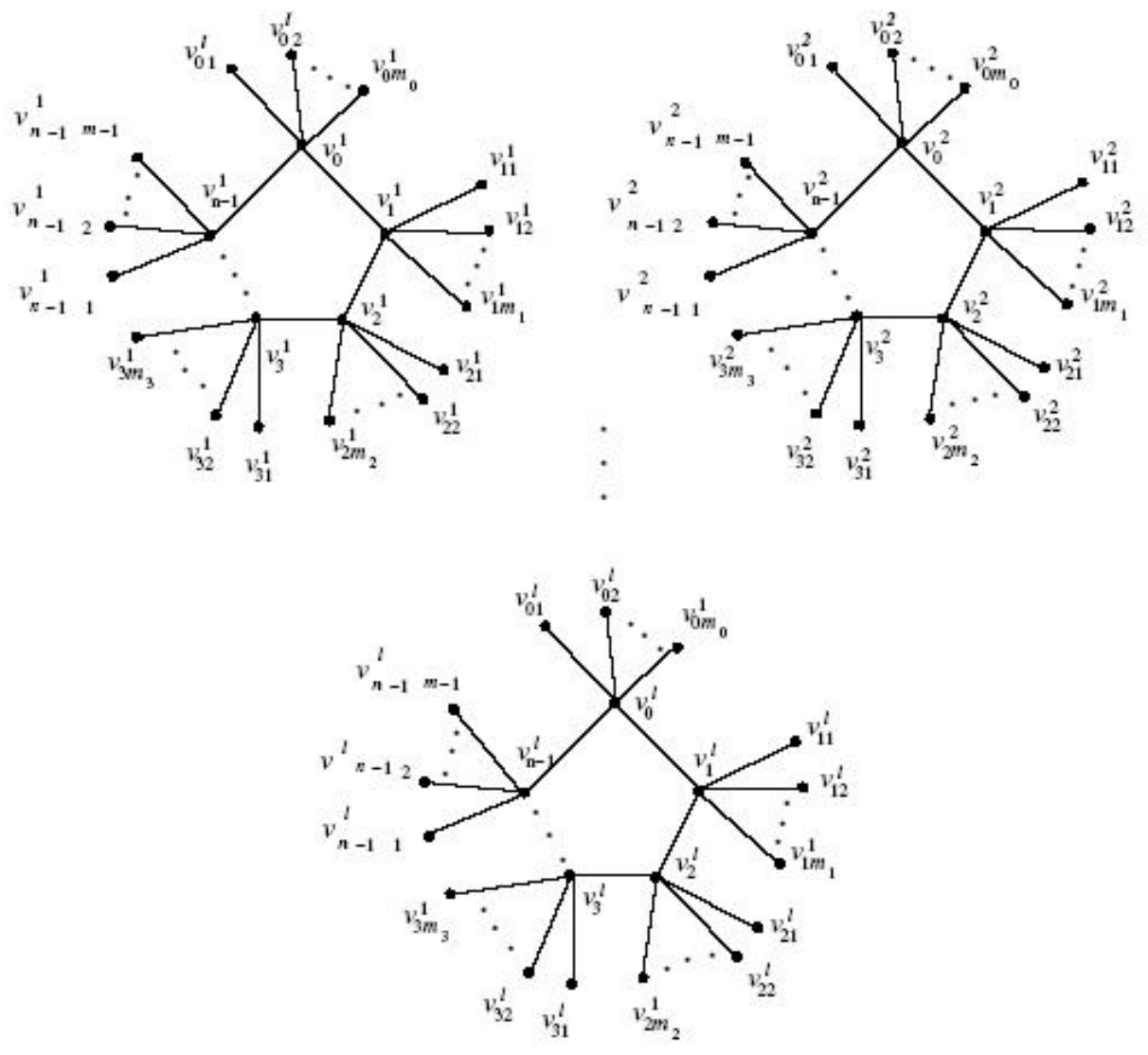

Figure 4.1: The graph $l A\left(m_{j}, n\right)$

Theorem 4.1. The graph $l A\left(m_{j}, n\right)$ is vertex-graceful for both $n$ and $l$ odd, $0 \leq i \leq n-1,1 \leq j \leq m_{i}$.

Proof. Consider the graph $G=l A\left(m_{j}, n\right)$ for both $n, l$ odd, $0 \leq i \leq$ $n-1,1 \leq j \leq m_{i}$. Let $v_{0}^{k}, v_{1}^{k}, v_{2}^{k}, \cdots, v_{n-1}^{k}$ be the vertices of the $k^{t h}$ copy of the cycle in $G$. Let $v_{i j}^{k}$ denote the pendant vertices of the $k^{\text {th }}$ copy such that $v_{i j}^{k}$ are adjacent to the vertices $v_{i}^{k}$ for $0 \leq i \leq n-1,1 \leq j \leq m_{i}, 1 \leq k \leq l$. It is easily verified that $G$ has $p=l\left(n+\sum_{i=1}^{n} m_{i}\right)$ vertices and $q=p$ edges. The required vertex-graceful labeling for vertices is defined as follows: 
Let $f: V \rightarrow\{1,2, \cdots, p\}$ be such that

$$
\begin{gathered}
\mathrm{f}\left(\mathrm{v}_{i}^{l}\right)=\left\{\begin{array}{c}
p(k-1) l+\frac{i}{2}+\sum_{a=1}^{\frac{i}{2}} m_{2 a-1}+1, \\
0 \leq i \leq n-1, i \text { even; } 1 \leq k \leq l . \\
\frac{p(k-1)}{l}+\frac{n-1}{2}+\sum_{a=1}^{\frac{i-1}{2}} m_{2 a-1}+\sum_{a=0}^{\frac{i-1}{2}} m_{2 a}+\frac{i+3}{2}, \\
1 \leq i \leq n-1, i \text { odd } ; 1 \leq k \leq l . \\
\mathrm{f}\left(\mathrm{v}_{i j}^{l}\right)=\left\{\begin{array}{c}
\frac{i-2}{2} \\
p(k-1) l+\frac{n-1}{2}+\sum_{a=1}^{\frac{n-1}{2}} m_{2 a-1}+\sum_{a=0} m_{2 a}+\frac{i}{2}+j+1, \\
0 \leq i \leq n-1, i \text { even; } 1 \leq j \leq m_{i} ; 1 \leq k \leq l .
\end{array}\right. \\
\frac{p(k-1)}{l}+\frac{i+1}{2}+\sum_{a=1}^{\frac{i-1}{2}} m_{2 a-1}+j, \\
1 \leq i \leq n-1, i \text { odd } ; 1 \leq j \leq m_{i} ; 1 \leq k \leq l .
\end{array}\right.
\end{gathered}
$$

The corresponding edge labeling is defined as follows:

Let $\quad \mathrm{A}=\left\{f^{*}\left(e_{i}^{k}\right): f^{*}\left(e_{i}^{k}\right)=\left(\frac{2 p(k-1)}{l}+\sum_{a=1}^{\frac{n-1}{2}} m_{2 a-1} \frac{n-1}{2}+2\right.\right.$

$\left.\left.\sum_{a=1}^{(i+1) \bmod n} m_{a-1}+(i+1) \bmod n\right)(\bmod q)\right\}$.

$\mathrm{B}=\left\{f^{*}\left(e_{i j}^{k}\right): f^{*}\left(e_{i j}^{k}\right)=\left(\frac{2 p(k-1)}{l}+\sum_{a=1}^{\frac{n-1}{2}} m_{2 a-1} \frac{n-1}{2}+\right.\right.$

$\left.\left.\sum_{a=0}^{i-1} m_{a}+i+j+2\right)(\bmod q)\right\}$.

It is clear that the vertex labels are distinct. Also it is easily verified that the edge labels sets are mutually disjoint and $A \cup B=\{0,1,2, \cdots, q-$ $1\}$. Therefore it follows that the induced mapping $f^{*}: E \rightarrow\{0,1,2, \cdots, q-$ $1\}$ is a bijection. Hence the graph $l A\left(m_{j}, n\right), 0 \leq i \leq n-1,1 \leq j \leq m_{i}$ is vertex-graceful for both $n$ and $l$ odd.

We leave the following problem as an open question.

Problem 4.2. Is it true that the graph $l A\left(m_{j}, n\right)$ is vertex-graceful when

(i) $n$ odd and $l$ even ?

(ii) $n$ even and $l$ even ?

(iii) $n$ even and $l$ odd ? 
Illustrative example of the labeling of $G$ of Theorem 4.1 is given in Figure 4.2.

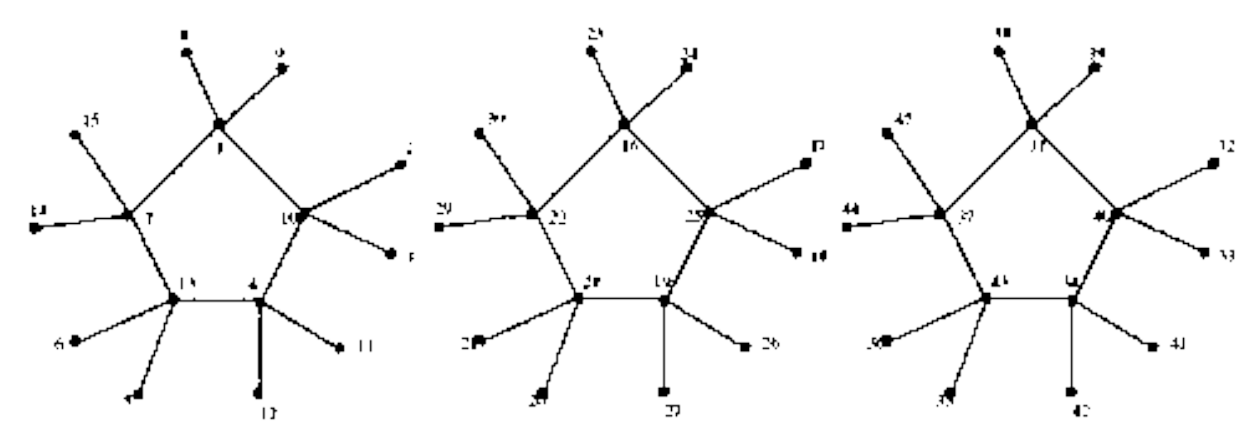

Figure 4.2: A vertex graceful labeled graph of $3 A(2,5), m_{1}=m_{2}=m_{3}=m_{4}=m_{5}=2$.

Remark 4.3. If $l=1$ in Figure 4.1, then the graph $G$ represents a actinia. Hence it follows from Theorem 4.1 that the graph $A\left(m_{j}, n\right)$ is vertexgraceful for any $m_{j}, 0 \leq j \leq n-1, n \geq 3$ with $n$ odd.

Theorem 4.4. The graph $A\left(m_{j}, n\right)$ is strong vertex-graceful for $n$ odd, $0 \leq i \leq n-1,1 \leq j \leq m_{i}$.

Proof. Consider the graph $G=A\left(m_{j}, n\right)$ for $n$ odd, $0 \leq i \leq n-1,1 \leq$ $j \leq m_{i}$. Let $v_{0}, v_{1}, v_{2}, \cdots, v_{n-1}$ be the vertices of the cycle in $G$. Let $v_{i j}$ denote the pendant vertices of $G$ such that $v_{i j}$ are adjacent to the vertices $v_{i}$ for $0 \leq i \leq n-1,1 \leq j \leq m_{i}$. It is easily verified that $G$ has $p=n+\sum_{i=0}^{n-1} m_{i}$ vertices and $q=p$ edges. The required strong vertex-graceful labeling for vertices is defined as follows:

Let $f: V \rightarrow\{1,2, \cdots, p\}$ be such that

$$
\begin{gathered}
\mathrm{f}\left(\mathrm{v}_{i}\right)=\left\{i 2+\sum_{a=1}^{\frac{i}{2}} m_{2 a-1}+1, \quad 0 \leq i \leq n-1, i\right. \text { even. } \\
\frac{n-1}{2}+\sum_{a=1}^{\frac{n-1}{2}} m_{2 a-1}+\sum_{a=0}^{\frac{i-1}{2}} m_{2 a}+\frac{i+3}{2}, \quad 1 \leq i \leq n-1, i \text { odd. } \\
\mathrm{f}\left(\mathrm{v}_{i j}\right)=\left\{\begin{array}{c}
\sum_{a=1}^{\frac{n-1}{2}} m_{2 a-1}+\frac{n-1}{2}+\sum_{a=0}^{\frac{i-2}{2}} m_{2 a}+\frac{i}{2}+j+1, \\
0 \leq i \leq n-1, i \text { even; } 1 \leq j \leq m_{i} .
\end{array}\right.
\end{gathered}
$$




$$
\begin{aligned}
& \sum_{a=1}^{\frac{i-1}{2}} m_{2 a-1}+\frac{i+1}{2}+j, \\
& \quad 1 \leq i \leq n-1, i \text { odd }, 1 \leq j \leq m_{i} .
\end{aligned}
$$

The corresponding edge labeling is defined as follows:

$$
\begin{aligned}
& \text { Let } \beta=\sum_{a=1}^{\frac{n-1}{2}} m_{2 a-1}+\frac{n-1}{2} \\
& \text { Let } A=\left\{f_{1}\left(e_{i}\right): f_{1}\left(e_{i}\right)=\beta+\sum_{a=1}^{(i+1) \bmod n} m_{a-1}+((i+1) \bmod n)+2\right\} \text {, } \\
& \text { where } e_{i}=v_{i} v_{(i+1) \bmod n}, 0 \leq i \leq n-1 \text {. } \\
& =\left\{\beta+m_{0}+3, \beta+m_{0}+m_{1}+4, \beta+m_{0}+m_{1}+m_{2}+5, \cdots, \beta+2\right\} . \\
& B=\left\{f_{1}\left(e_{i j}\right): f_{1}\left(e_{i j}\right)=\beta+\sum_{a=0}^{i-1} m_{a}+i+j+2\right\}, \\
& \text { where } e_{i j}=v_{i} v_{i j}, 0 \leq i \leq n-1,1 \leq j \leq m_{i} \text {. } \\
& =\left\{\beta+3, \beta+4, \cdots, \beta+m_{0}+2 ; \beta+m_{0}+4 ; \beta+m_{0}+5, \cdots, \beta+m_{0}+\right. \\
& m_{1}+3, \cdots ; \beta+m_{0}+m_{1}+\cdots, m_{n-2}+n+2, \beta+m_{0}+m_{1}+\cdots, m_{n-2}+ \\
& \left.n+3, \cdots, \beta+m_{0}+m_{1}+\cdots, m_{n-2}+n+1+m_{n-1}\right\} \text {. }
\end{aligned}
$$

It is clear that the vertex labels are distinct. Also it is easily verified that the edge labels sets are mutually disjoint and $A \cup B=\{\beta+2, \beta+$ $\left.3, \cdots, \beta+m_{0}+m_{1}+\cdots,+m_{n-2}+m_{n-1}+n+1\right\}$. Therefore the induced edge labels of $G$ have $q$ consecutive values. Hence the graph $G$ is strong vertex-graceful. 
Illustrative examples of the labeling of Theorem 4.4 is given in Figure 4.3 .

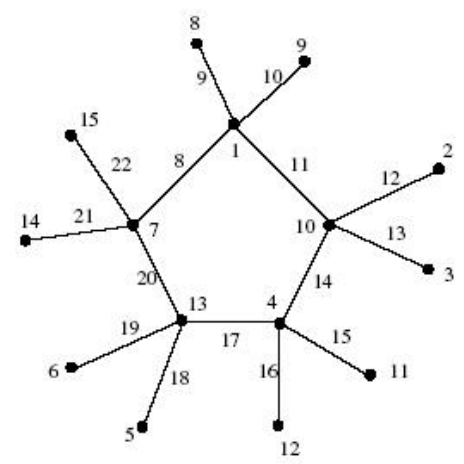

Figure 4.3: Strong vertex-graceful labeled graph of $A(2,5)$.

\section{References}

[1] D. Acharya, S. Arumugam, and A. Rosa, Labeling of Discrete Structures and Applications, Narosa Publishing House, New Delhi, (2008).

[2] P. Bahl, S. Lake, and A. Wertheim, Gracefulness of Families of Spiders, Involve, 3, pp. 241-247, (2010).

[3] S. Bloom and D. F. Hsu, On graceful digraphs and a problem in network addressing, Congr. Numer., 35, pp. 91-103, (1982).

[4] S. Bloom and D. F. Hsu, On graceful directed graphs that are computational models of some algebraic systems, Graph Theory with Applications to Algorithms and Computers, Ed. Y. Alavi, Wiley, New York, (1985).

[5] G. Chartrand and P. Zhang, An Introduction to Graph Theory, Tata McGrawHill Edition, (2006).

[6] J. A. Gallian, A Dynamic Survey of Graph Labeling, The Electronic. J. Combin., DS17, pp. 1-384, (2014).

[7] S. W. Golomb, How to number a graph, Graph Theory and Computing, R. C.Read, ed., Academic Press, New York, pp. 23-37, (1972). 
[8] F. Harary, Graph Theory, Addison-Wesley, (1969).

[9] F. Hsu and A. D. Keedwell, Generalized complete mappings, neofields, sequenceable groups and block designs, I, Pacific J. Math., 111, pp. 317-332, (1984).

[10] F. Hsu and A. D. Keedwell, Generalized complete mappings, neofields, sequenceable groups and block designs, II, Pacific J. Math., 117, pp. 291-312, (1985).

[11] J. Jeba Jesintha and G. Sethuraman, A new class of graceful rooted trees, J. Discrete Math. Sci. Cryptogr., 11, pp. 421-435, (2008).

[12] A. Rosa, On certain valuations of the vertices of a graph, Theory of Graphs, Gordon and Breach, N. Y. and Dunod Paris, pp. 349-355, (1967).

[13] P. Selvaraju, P. Balaganesan and J. Renuka, V. Balaji, On Vertexgraceful Labeling, Bulletin of Kerala Mathematics Association, 9, pp. 179-184, (2012).

[14] P. Selvaraju, P. Balaganesan and J. Renuka, Vertex-graceful graph $c_{2 k} \cup$ $C_{2 k+1}$, European Journal of Scientific and Research, 97, pp. 192-196, (2013).

[15] P. Selvaraju, P. Balaganesan and J. Renuka, Vertex-graceful Labeling of Some Path Related Graphs, International J. Math. Combin., 3, pp. 44-49, (2013).

[16] P. Selvaraju, P. Balaganesan and J. Renuka and M. L. Suresh, Vertexgraceful Labeling of $C_{i} \cup C_{j} \cup C_{l}$, Applied Mathematical Sciences, 8 (82), pp. 4047-4052, (2014).

[17] P. Selvaraju, P. Balaganesan and J. Renuka and M. L. Suresh, Harmonious and Vertex-graceful Labeling of Path and Star Related Graphs, International Journal of Pure and Applied Mathematics, 93, pp. 501509, (2014).

[18] Sin-min Lee, Y. C. Pan and Ming Chen Tsai, On vertex-graceful graph $(p, p+1)$ - graphs, Congressus Numerantium 172, pp. 65-78, (2009).

[19] W. C. Shiu, P. C. B. Lam, Super-edge-graceful labelings of multi-level wheel graphs, fan graphs and actinia graphs, Congressus Numerantium 174, pp. 49-63, (2005). 
A. P. Santhakumaran

Department of Mathematics

Hindustan Institute of Technology and Science

Chennai 603103, Tamil Nadu,

India

e-mail : apskumar1953@gmail.com

and

P. Balaganesan

Research Scholar

Department of Mathematics

Hindustan Institute of Technology and Science

Chennai 603103, Tamil Nadu,

India

e-mail : balaganesanpp@gmail.com 